\title{
A Priori Testing of Flamelet Generated Manifolds for Turbulent Partially Premixed Methane/Air Flames
}

\author{
W. J. S. Ramaekers • J. A. van Oijen • L. P. H. de Goey
}

Received: 7 September 2008 / Accepted: 8 May 2009 / Published online: 26 May 2009

(C) The Author(s) 2009. This article is published with open access at Springerlink.com

\begin{abstract}
To reduce high computational cost associated with simulations of reacting flows chemistry tabulation methods like the Flamelet Generated Manifold (FGM) method are commonly used. However, $\mathrm{H}_{2}, \mathrm{CO}$ and $\mathrm{OH}$ predictions in RANS and LES simulations using the FGM (or a similar) method usually show a substantial deviation from measurements. The goal of this study is to assess the accuracy of low-dimensional FGM databases for the prediction of these species in turbulent, partially-premixed reacting flows. It will be examined to what extent turbulent, partially-premixed jet flames can be described by FGM databases based on premixed or counterflow diffusion flamelets and to what extent the chosen molecular transport model for the flamelet influences the accuracy of species mass fraction predictions in CFD-simulations. For LES and RANS applications a model that accounts for subgrid fluctuations has to be added introducing additional errors in numerical results. A priori analysis of FGM databases enables the exclusion of numerical errors (scheme accuracy, convergence) that occur in CFD simulations as well as the exclusion of errors originating from subgrid modeling assumptions in LES and RANS. Four different FGM databases are compared for $\mathrm{H}_{2} \mathrm{O}, \mathrm{H}_{2}, \mathrm{CO}, \mathrm{CO}_{2}$ and $\mathrm{OH}$ predictions in Sandia Flames $\mathrm{C}$ to F. Species mass fractions will be compared to measurements directly and conditioned on mixture fraction. Special attention is paid to the representation of experimentally observed differential diffusion effects by FGM databases.
\end{abstract}

Keywords Turbulent combustion • Partially-premixed • Reduced chemistry • Flamelet Generated Manifold

W. J. S. Ramaekers $(\bowtie)$ · J. A. van Oijen · L. P. H. de Goey

Section Combustion Technology, Department of Mechanical Engineering,

Eindhoven University of Technology, PO Box 513, 5600 MB Eindhoven, The Netherlands

e-mail:w.j.s.ramaekers@tue.nl 


\section{Introduction}

Accurate predictions of pollutant emissions in engineering combustion appliances pose a serious challenge for combustion engineers. Computational cost associated with solving the very stiff system of transport equations, due to the large range of timescales, is very high. If additionally detailed chemistry is required for accurate predictions of species like $\mathrm{H}_{2}, \mathrm{CO}$ and $\mathrm{OH}$ numerical simulations of reactive flow become impractical or even impossible. This is especially true for DNS and highresolution LES simulations where solving the flow field is already very demanding in terms of computational resources. On the other hand DNS and LES methods are highly desired for the simulation of turbulent reacting flows because turbulent fluctuations are, at least to a great extent, are resolved and for their substantial influence on combustion chemistry less modeling assumptions have to be made. For DNS and LES simulations of reacting, high Reynolds number flows methods are desired to reduce the computational cost associated with detailed chemistry. These methods would enable the simulation of real-life engineering combustion devices and thereby allow the minimization of harmful emissions and fuel consumption.

To reduce the computing resources required for simulation of finite-rate chemical kinetics chemical reduction techniques are commonly applied. Tabulation methods form one class of methods within chemical reduction techniques and their advantage is that no elementary reactions or species are removed from the chemical reaction mechanism. They generate a detailed database for specific well-defined conditions. In this database thermo-chemical variables are stored as a function of a small number of controlling variables. Several methodologies to tabulate complex chemistry have been developed: tabulation can be done on-line like the well-known In Situ Adaptive Tabulation (ISAT) [1] or in advance of the CFD simulation. Some well-known examples of in advance tabulation methods are the Intrinsic Low Dimensional Manifold (ILDM) [2] and the more recent Reaction Diffusion Manifold (REDIM) $[3,4]$ method and the Flamelet Prolongated ILDM (FPI) [5] or Flamelet Generated Manifold (FGM) [6] reduction method. In the FPI or FGM method it is assumed that trajectories in composition space in turbulent flames closely resemble trajectories in composition space in laminar, one-dimensional flames called flamelets [7]. In this study the FGM reduction method will be used for representation of laminar combustion chemistry since it allows the use of (unsteady) premixed flamelets, (unsteady) counterflow diffusion flamelets or homogenous reactor computations (ignition flamelets).

Previously Chen et al. [8] showed that a chemistry database, generated from perfectly stirred reactor computations, overestimated CO mass fractions in rich parts of a methane/air diffusion flame. Pitsch and Steiner [9] performed LES simulations of Sandia Flame D using a Lagrangian Flamelet Model (LFM) incorporating diffusion in mixture fraction direction. A database was generated and parameterized on mixture fraction $(Z)$ and scalar dissipation rate $(\chi)$. To incorporate subgrid influences on chemical kinetics a Presumed PDF method using a marginal $\beta$-PDF for $Z$ is used. $\chi$ is subsequently computed from the $Z$ field. Conditional mean $\mathrm{CO}_{2}, \mathrm{CO}, \mathrm{H}_{2} \mathrm{O}, \mathrm{H}_{2}$ and $\mathrm{OH}$ mass fractions were presented of which $\mathrm{CO}_{2}$ was very well predicted. The conditional mean $\mathrm{H}_{2} \mathrm{O}$ mass fraction was underpredicted for $Z$ values larger than the stoichiometric value while both $\mathrm{CO}$ and $\mathrm{H}_{2}$ were overestimated under fuel-rich conditions. Especially at $x=15 D$ the maximum $\mathrm{OH}$ mass fraction was significantly 
overestimated. It was argued that premixing of the fuel with air occurred to fast causing combustion reactions to take place too early. This, in turn, led to a slight overprediction in formation of $\mathrm{H}_{2} \mathrm{O}$ and the intermediates $\mathrm{H}_{2}$ and $\mathrm{CO}$ in the fuelrich region. The overestimation of mixing in the flow field is here said to cause the discrepancy in species mass fractions predictions. Vervisch et al. [10] simulated Sandia Flame D using a combination of FPI-/diff usion flamelet approach. During the simulation chemical source terms were retrieved from the FPI-database (based on premixed flamelets) which was parameterized on $Z$ and a reaction progress variable $(\mathcal{Y})$. Species mass fractions were interpolated a posteriori from the diffusion flamelet database. To model the influence of subgrid fluctuations of combustion chemistry a PPDF-closure method was adopted using a marginal $\beta$-PDF for both $Z$ and $\mathcal{Y}$. Only species mass fractions results for $\mathrm{CO}$ were shown in mixture fraction space for Sandia Flame D at heights $x=3.0 D, x=15 D$ and $x=60 D$ from the inlet plane. At $x=15 D$, which is by far the most interesting because information is available for the entire range in mixture fraction, conditional mean $\mathrm{CO}$ mass fractions were slightly overestimated on the fuel-rich side and a significant underestimation occurred at $Z \approx 0.4$. The same deviations from measurements were observed in conditional mean $\mathcal{Y}$ values; these deviations were attributed to various factors of which one was the chemical scheme in combination with the used (premixed flamelet based) FPI tabulation method. Fiorina et al. [11] analyzed how well a FPI database, also parameterized on $Z$ and $\mathcal{Y}$, represented complex chemistry for methane/air premixed, partially-premixed and diffusion flames. For the premixed flame it was found that the FPI database reproduced results from detailed chemistry very well. However, for the partially-premixed and diffusion flames significant differences for temperature, $\mathrm{CO}_{2}$ and $\mathrm{CO}$ mass fractions were observed between the FPI predictions and detailed chemistry for fuel-rich conditions. Apparently a premixed flameletbased database cannot represent chemical kinetics accurately for partially-premixed and diffusion flames in fuel-rich conditions. Vreman et al. [12] performed LES simulations of Sandia Flames D and F using a premixed flamelet-based and a counterflow diffusion flamelet-based FGM database. It was found that $\mathrm{CO}_{2}, \mathrm{H}_{2} \mathrm{O}$, $\mathrm{CH}_{4}$ and $\mathrm{O}_{2}$ mass fractions were well-predicted but $\mathrm{CO}, \mathrm{H}_{2}$ and $\mathrm{OH}$ mass fraction were predicted less satisfactory although the counterflow diffusion flamelet-based FGM yielded significantly better results than the premixed flamelet-based FGM. Although Vreman et al. [12] found that the inclusion of modeled subgrid variances of mixture fraction and progress variable as additional entries to the manifold had only small effects on the simulation of both Sandia Flame D and F, the question remains whether the error in $\mathrm{CO}, \mathrm{H}_{2}$ and $\mathrm{OH}$ mass fractions originates from the tabulated chemistry or from the LES simulation.

In LES or RANS simulations of turbulent reacting flow using tabulated chemistry combined with a subgrid model, three main origins of errors can be distinguished. The first origin of errors can be attributed to an inappropriate representation of chemical kinetics by the tabulated chemistry, subsequently the subgrid modeling assumptions used in transport equations can be the origin of inaccuracies in numerical simulations. The third class of errors are made during the actual CFD simulation: the accuracy of the used numerical schemes and convergence of the results.

The objective of this paper is to assess the accuracy of representation of chemical kinetics by low-dimensional FGM databases for turbulent partially-premixed jet flames. In order to eliminate errors originating from CFD simulations, an a priori 
analysis of FGM databases is performed in which FGM databases are directly compared to experimental results. It is investigated to what extent the used flamelet structure type (premixed or counterflow diffusion flamelets) and the molecular transport model (multi-component diffusion or unit Lewis numbers) should resemble the characteristics of the analyzed turbulent flame to yield an accurate representation of combustion chemistry. The influence of the flamelet type and the molecular transport model on the accuracy of mass fraction predictions will be assessed for Sandia Flames C, D, E and F [13], which exhibit behavior ranging from moderately turbulent (Flame $\mathrm{C}, R e=13.400$ ) to heavily turbulent with local extinction and reignition (Flame F, $R e=44.800$ ). More specifically, the focus will be on predictions of $\mathrm{CO}, \mathrm{H}_{2}$ and $\mathrm{OH}$ since these are still hard to predict accurately.

In the next section the generation of the FGM databases will be discussed for both premixed- and counterflow diffusion flamelet-based databases. This is followed by the analysis of the generated FGM databases which is split up into three parts: in the first part a direct comparison between measured and predicted species mass fractions and in the second part an analysis conditioned on $Z$ to gain more insight on the underlying physics. In the third part an analysis of which type of FGM database can adequately describe differential diffusion effects will be performed; this analysis is performed to determine the appropriate molecular transport model to be used in FGM databases for current test cases. The paper ends with a recommendation which type of FGM database should be used to simulate the Sandia Flames and the accuracy of $\mathrm{CO}, \mathrm{H}_{2}$ and $\mathrm{OH}$ mass fraction predictions when using the best-suited FGM database.

\section{Generation of FGM Databases}

Before FGM databases can be compared with experimental data they first have to be generated. In this section the equations that describe the flamelet solutions of which FGM's are composed are dealt with. Boundary conditions for the different types of flamelets to be used and methods to construct FGM's from multiple flamelet solutions are subsequently discussed.

\subsection{Parametrization of FGM database}

For this study, partially-premixed combustion parameters are mapped on two control variables describing mixing (mixture fraction $Z$ ) and reaction progress (reaction progress variable $\mathcal{Y})$ :

$$
\phi=\phi(Z, \mathcal{Y})
$$

in which $\phi$ can denote any thermo-chemical variable. The mixture fraction $Z$ is defined by Bilger [14] which was modified by Barlow et al. [15] by removing the oxygen terms and thereby making the mixture fraction less sensible to experimental noise and interference from laser-induced fluorescence [15-17]. The mixture fraction definition proposed by Barlow et al. reads:

$$
Z=\frac{2 M_{H}^{-1}\left[\mathcal{Z}_{H}-\mathcal{Z}_{H, 2}\right]+0.5 M_{C}^{-1}\left[\mathcal{Z}_{C}-\mathcal{Z}_{C, 2}\right]}{2 M_{H}^{-1}\left[\mathcal{Z}_{H, 1}-\mathcal{Z}_{H, 2}\right]+0.5 M_{C}^{-1}\left[\mathcal{Z}_{C, 1}-\mathcal{Z}_{C, 2}\right]}
$$


in which $M_{j}$ denotes the molar mass of an element $j, \mathcal{Z}_{j}$ denotes the mass fraction of an element and subscripts $H, C, 1$ and 2 refer to hydrogen, carbon and the fueland oxidizer stream, respectively. The reaction progress variable, which has to be monotonous in both lean and rich mixtures in order to facilitate an unambiguous determination of dependent variables, for the Sandia Flames is defined as:

$$
\mathcal{Y}=\alpha_{1} Y_{\mathrm{CO}_{2}}+\alpha_{2} Y_{\mathrm{H}_{2} \mathrm{O}}+\alpha_{3} Y_{\mathrm{H}_{2}}
$$

in which $\alpha$ denotes a weight factor for each component present in $\mathcal{Y}$ and $Y_{\mathrm{i}}$ denotes a species mass fraction. Values for $\alpha_{i}$ are not trivial; it was found that values for $\alpha$ equal to unity divided by the molar mass of the corresponding species ensured a monotonous increasing $\mathcal{Y}$ for all flamelets used.

\subsection{Flamelet equations}

The flamelet equations [6] form a set of specific transport equations for onedimensional reacting flows describing conservation of mass, species and enthalpy:

$$
\begin{aligned}
\frac{\partial(\rho u)}{\partial x} & =-\rho K \\
\frac{\partial\left(\rho u Y_{\mathrm{i}}\right)}{\partial x} & =\frac{\partial}{\partial x}\left(\rho \mathbf{V}_{\mathrm{i}} Y_{\mathrm{i}}\right)+\dot{\omega}_{i}-\rho K Y_{\mathrm{i}} \\
\frac{\partial(\rho u h)}{\partial x} & =\frac{\partial}{\partial x}\left(\frac{\lambda}{c_{p}} \frac{\partial h}{\partial x}+\sum_{i=1}^{\mathrm{N}_{\mathrm{s}}} h_{i}\left(\rho \mathbf{V}_{\mathrm{i}} Y_{\mathrm{i}}-\frac{\lambda}{c_{p}} \frac{\partial Y_{\mathrm{i}}}{\partial x}\right)\right)-\rho K h
\end{aligned}
$$

in which $x, \rho, u, K$ denote the physical coordinate perpendicular to the flame, the mixture density, the velocity of the gas mixture and the flame stretch [18] respectively. $Y_{\mathrm{i}}, \mathbf{V}, \dot{\omega}, \lambda, c_{p}$ and $\mu$ denote the mass fraction of species $i$, the diffusion velocity, the chemical production rate, the thermal conductivity, the specific heat at constant pressure and the dynamic viscosity, respectively. $\mathrm{N}_{\mathrm{s}}$ denotes the total number of species present in the used reaction mechanism. Total enthalpy $h$ is defined by:

$$
h(T)=\sum_{i=1}^{\mathrm{N}_{\mathrm{s}}} Y_{i}\left[h_{i}^{0}+\int_{T^{0}}^{T} c_{p, i}\left(T^{*}\right) d T^{*}\right]
$$

The low-Mach approximation is applied to prohibit acoustic waves propagating though the computational domain:

$$
p^{0}=\rho R T \sum_{i=1}^{\mathrm{N}_{\mathrm{s}}}\left(Y_{\mathrm{i}} / M_{i}\right)
$$

in which $p^{0}$ denotes the background pressure, $R$ denotes the universal gas constant and $M_{i}$ denotes the molecular mass of species $i$. For counterflow diffusion flames an additional transport equation for the unknown stretch field $K$ has to be solved:

$$
\frac{\partial(\rho u K)}{\partial x}=\frac{\partial}{\partial x}\left(\mu \frac{\partial K}{\partial x}\right)-\rho K^{2}+\rho_{2} a^{2}
$$


in which $a$ denotes the applied strain rate $[1 / \mathrm{s}]$ at the oxidizer side and subscript 2 refers to the oxidizer stream. This transport equation has been derived by DixonLewis [19]; here the formulation for two-dimensional cartesian geometries has been adopted.

For molecular transport both multi-component diffusion including the Soret- and Dufour effect and the unit Lewis number assumption are used in this study. Weakly turbulent combustion tends to be better described with the use of a multi-component diffusion transport model due to the dominance of molecular diffusive processes over turbulent redistribution. When the flow becomes more turbulent, redistribution by turbulent eddies, which do not discriminate between different species to be mixed, will start to dominate molecular diffusion. It is therefore investigated whether weakly turbulent combustion is better described by a FGM using a multi-component diffusion transport model and with increasing turbulence intensity combustion is increasingly well described by a FGM using a unit Lewis number transport model. The unit Lewis number assumption has the advantage that it allows the reduction of (5) and (6) to more simple formulations:

$$
\begin{aligned}
& \frac{\partial\left(\rho u Y_{\mathrm{i}}\right)}{\partial x}=\frac{\partial}{\partial x}\left(\frac{\lambda}{c_{p}} \frac{\partial Y_{\mathrm{i}}}{\partial x}\right)-\rho K Y_{\mathrm{i}}+\dot{\omega}_{i} \\
& \frac{\partial(\rho u h)}{\partial x}=\frac{\partial}{\partial x}\left(\frac{\lambda}{c_{p}} \frac{\partial h}{\partial x}\right)-\rho K h
\end{aligned}
$$

The set of governing equations describing either premixed- or non-premixed flamelets is solved by the fully implicit solver CHEM1D [20] developed at the Eindhoven University of Technology.

\subsection{Boundary conditions for the flamelet equations}

When FGM's are to be constructed from unstrained steady premixed flamelets, (4), (5) and (6) are solved. $K$ equals zero everywhere and (9) thereby is redundant. Equation (7) and the equation of state, (8), close the set of equations. Boundary conditions for premixed flamelets are:

$$
\begin{aligned}
u(x \rightarrow-\infty) & =s_{\mathrm{L}}\left(Z_{u}\right) \\
Y_{\mathrm{i}}(x \rightarrow-\infty) & =Z_{u} Y_{\mathrm{i}, 1}+\left(1-Z_{u}\right) Y_{\mathrm{i}, 2} \\
h(x \rightarrow-\infty) & =Z_{u} h_{1}+\left(1-Z_{u}\right) h_{2}
\end{aligned}
$$

in which subscript $u$ denotes the unburnt mixture, $s_{\mathrm{L}}$ denotes the adiabatic flame propagation velocity and is an eigenvalue of the system of equations. Subscripts 1 and 2 refer to the fuel- and oxidizer stream, respectively. The parameter of this system is the stoichiometry of the fresh mixture denoted by the mixture fraction $Z_{u}$. The translational degree of freedom is removed by choosing a fixed temperature at $x=0$. Each flamelet contained 200 gridpoints and the premixed flamelet based FGM databases contains 450 flamelets between the numerical flammability limits; for the Sandia Flames the lower numerical flammability limit was found at $Z=0.1$ and the upper flammability limit was still not reached at $Z=1$. Beyond flammability limits thermo-chemical variables are linearly interpolated between the leanest flamelet 
and pure oxidizer and the richest flamelet and pure fuel, respectively. Chemical equilibrium has been calculated and explicitly added to all flamelets to ensure equilibrium mass fractions for $\mathrm{CO}$ and $\mathrm{CO}_{2}$. When multi-component diffusion is employed, $Z$ is not constant in a premixed flamelet due to differential diffusion effects. To account for this phenomena the FGM database is interpolated onto a $Z-\mathcal{Y}$ grid which is equidistant in both directions.

When FGM's are constructed from strained counterflow diff usion flamelets, (4), (5), (6) and (9) are solved. Equations of state, (7) and (8), again close the set of equations. Boundary conditions for strained counterflow diffusion are:

$$
\begin{aligned}
Y_{\mathrm{i}}(x \rightarrow-\infty) & =Y_{\mathrm{i}, 1} \\
h(x \rightarrow-\infty) & =h_{1} \\
Y_{\mathrm{i}}(x \rightarrow+\infty) & =Y_{\mathrm{i}, 2} \\
h(x \rightarrow+\infty) & =h_{2} \\
K(x \rightarrow+\infty) & =a
\end{aligned}
$$

in which subscripts 1 and 2 again refer to the fuel- and oxidizer stream, respectively. The parameter of this system is the applied strain rate $a$, which is defined at the oxidizer side $(x=\infty)$. The translational degree of freedom is removed by choosing the stagnation plane at $x=0$. Please note that counterflow diffusion flamelets are computed in physical space instead of $Z$-space. This has the advantage that no assumption has to be made for the profile of the scalar dissipation rate $\chi$, which occurs when (5) and (6) are transformed to $Z$-space. Because counterflow diffusion flamelets extinguish at a limiting strain rate the region in $Z-\mathcal{Y}$ space between the maximum strain rate and the mixing limit cannot be spanned by steady counterflow diffusion flamelets. Beyond the extinction strain rate time-dependent solutions of (4), (5), (6) and (9) are used: this results in a natural continuation of the profiles in $Z-\mathcal{Y}$ space. The most strained steady counterflow diffusion flamelet served as an initial solution and subsequently the strain-rate has been increased by unity to establish quenching of the flame. The time-dependent solution of the unsteady equations, which is treated as a series of solutions, is tracked until the solution equals the mixing limit. For a quenching counterflow diffusion flamelet $\mathcal{Y}$ decreases: the use of these unsteady flamelets does not require an additional control variable. For the domain spanned by steady flamelet solutions the strain rate has been varied between $a=2$ [1/s] and the extinction strain rate: $a=657$ [1/s] for a multi-component diffusion transport model and $a=978[1 / \mathrm{s}]$ for a unit Lewis number transport model for the Sandia Flames. Each counterflow diffusion flamelet contained 250 gridpoints and the counterflow diffusion flamelet-based FGM databases contains 150 steady and 75 unsteady flamelets. The flamelet solutions are interpolated onto a $Z-\mathcal{Y}$ grid which is equidistant in both directions.

All FGM databases used in this study have a $201 \times 201(Z \times \mathcal{Y})$ equidistant discretization and data has been retrieved from these databases using linear interpolation. As an example $\mathrm{H}_{2} \mathrm{O}$ and $\mathrm{OH}$ as retrieved from the FGM-DL database are shown in Fig. 1. For all flamelets detailed chemistry is modeled by the GRI 3.0 reaction mechanism [21]. Fuel and oxidizer compositions have been taken from Liu et al. [22]. 

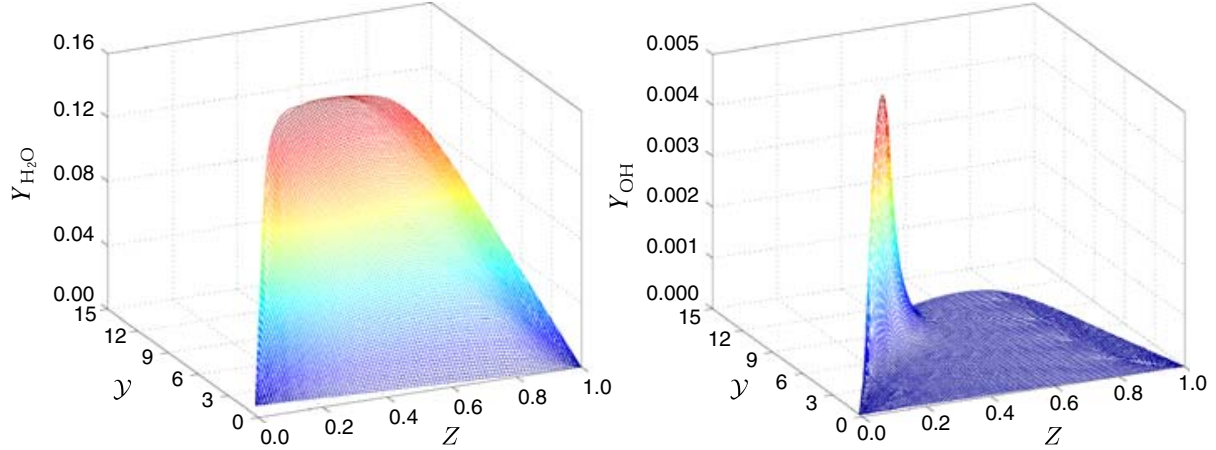

Fig. $1 \mathrm{H}_{2} \mathrm{O}$ and $\mathrm{OH}$ mass fractions as retrieved from the FGM database based on counterflow diffusion flames using unit Lewis numbers for all species (FGM-DL); every third entry for $Z$ and $\mathcal{Y}$ is shown. (Color in digital version only)

\section{A Priori Validation of FGM Databases}

The availability of extensive experimental data on Sandia Flames C, D, E and F by Barlow et al. [13] allows a priori validation of FGM databases. The experimental data consists of several thousands of instantaneous species mass fraction measurements at different heights and radial positions in all four flames. For every instantaneous measurement the reconstructed $Z$ and $\mathcal{Y}$ are computed according to (2) and (3); resulting scatter plots are shown in Fig. 2 for Sandia Flame C and F. Using these reconstructed values for $Z$ and $\mathcal{Y}$, corresponding thermochemical data can be retrieved from the FGM database. Subsequently experimentally observed averaged species mass fractions, indicated by $Y_{\mathrm{i}}^{\mathrm{EXP}}$, can be compared to averaged species mass fractions retrieved from the FGM database which are indicated by $Y_{\mathrm{i}}^{\mathrm{FGM}}$. It must be remarked that only measurement data from nine species, $\mathrm{O}_{2}, \mathrm{CH}_{4}, \mathrm{~N}_{2}, \mathrm{H}_{2} \mathrm{O}, \mathrm{CO}_{2}$, $\mathrm{H}_{2}, \mathrm{CO}, \mathrm{OH}$ and $\mathrm{NO}$, is available with measurement uncertainties equal to $0.4 \%$, $0.5 \%, 3 \%, 4 \%, 4 \%, 10 \%, 10 \%, 10 \%$ and $15 \%$ respectively. The sum of all measured mass fractions is less than $0.2 \%$ away from unity for all instantaneous measurements
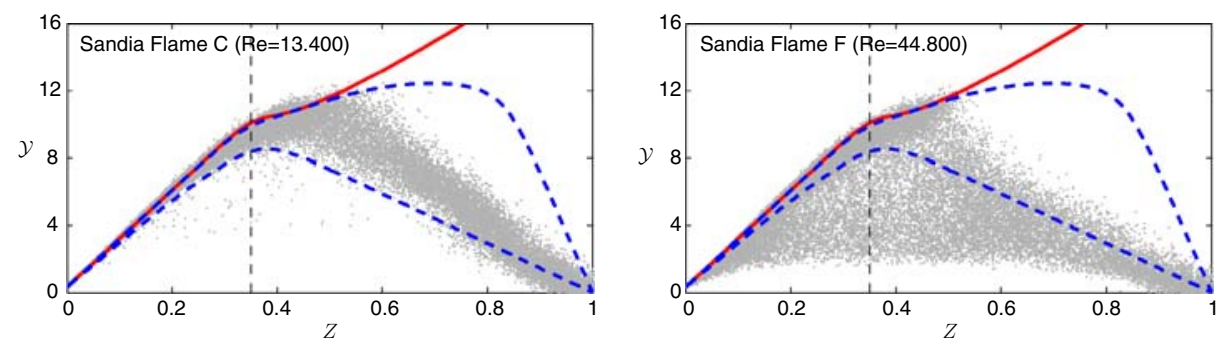

Fig. 2 Reconstructed $(Z, \mathcal{Y})$ scatter plots for Flame $\mathrm{C}$ and Flame $\mathrm{F}$. The vertical dashed black line indicates the stoichiometric mixture fraction. The solid red line indicates chemical equilibrium and the upper and lower dashed blue lines represent two counterflow diffusion flamelets with a strain rate equal to $2[1 / \mathrm{s}]$ and equal to the extinction strain rate $a_{\text {ext }}$ respectively. (Color in digital version only) 
at all probed locations, implying that mass fractions of species which have not been measured do not have a significant influence on the reconstructed value for $Z$ and $\mathcal{Y}$.

To allow the validation of a laminar combustion chemistry database the spatial resolution of measurements must be significantly smaller than the chemical length scale present in the turbulent flame. Spatial resolution of measurements has been estimated to be approximately $750 \mu \mathrm{m}$ [16, 23, 24]. A quick estimation of the chemical length scale tells us that it is of the same order of magnitude as the probe resolution:

$$
L_{\text {chem }} \propto \sqrt{\frac{D}{a}}=\sqrt{\frac{\mathcal{O}\left(10^{-5}\right)}{\mathcal{O}\left(10^{2}\right)}}=\mathcal{O}\left(10^{-3}\right)[\mathrm{m}] \sim L_{\text {probe }}
$$

In the discussion at the end of Barlow et al. [16] it is argued that spatial averaging over the probe volume has very limited influence on measured scalar quantities. For this study it is therefore assumed that spatial averaging over the probe volume is of sufficiently small influence to be neglected.

In this section results of a priori analysis of FGM databases for predictions of $\mathrm{H}_{2} \mathrm{O}$, $\mathrm{H}_{2}, \mathrm{CO}_{2}, \mathrm{CO}$ and $\mathrm{OH}$ will be discussed of which $\mathrm{H}_{2}, \mathrm{CO}$ and $\mathrm{OH}$ will be treated more in-depth. Four different FGM databases will be compared as stated in Table 1. Scatter plots will be presented to gain a global insight on the performance of different types of FGM databases; subsequently conditional (on mixture fraction) figures will give more insight to explain underlying physical phenomena.

\subsection{Direct comparison of measured with predicted mass fractions}

Direct comparison of experimentally observed with predicted mass fraction yield a quick, but not fully detailed, insight on the predictive accuracy of a FGM database. To allow quantification of the accuracy a definition for the error is introduced:

$$
\epsilon_{i}=\frac{\sqrt{\frac{1}{N} \sum_{k=1}^{N}\left(Y_{\mathrm{i}}^{\mathrm{EXP}}-Y_{\mathrm{i}}^{\mathrm{FGM}}\right)^{2}}}{\frac{1}{N} \sum_{k=1}^{N} Y_{\mathrm{i}}^{\mathrm{EXP}}}
$$

which equals the RMS-value of a species $i$ its mass fraction normalized by its average experimentally observed mass fraction. The definition contains a summation over instantaneous measurements; here the summation has been taken over all heights and radial positions for a specific flame. Differences between measured and predicted values can be caused by either measurement uncertainties and/or discrepancies between the experimentally observed- and tabulated chemistry. Measurement uncertainties cause both a spreading in measurement data and, subsequently, an inaccu-

Table 1 Designations for the four different FGM databases used in this study

\begin{tabular}{lll}
\hline Designation & Flamelet type & Molecular transport \\
\hline FGM-PL & Premixed & Unit Lewis numbers \\
FGM-PC & Premixed & Multi-component diffusion \\
FGM-DL & Counterflow diffusion & Unit Lewis numbers \\
FGM-DC & Counterflow diffusion & Multi-component diffusion \\
\hline
\end{tabular}


racy for $Z$ and $\mathcal{Y}$ which are reconstructed according to (2) and (3). An inaccuracy in reconstructed $Z$ and $\mathcal{Y}$ implies that data is retrieved from a not-exactly-right location in the FGM database. Discrepancies between the experimentally observed- and tabulated chemistry introduces a third origin for differences between instantaneous measurements and corresponding data from FGM databases. In order to minimize differences between (instantaneous) measurements and predictions, measurement uncertainties need to minimized and the type of FGM database should be tuned to the type of flame which is to be simulated. For averaged values the dispersion of instantaneous measurements introduces an additional error when tabulated thermochemical variables exhibit a curved profile in $Z-\mathcal{Y}$ space. This error can be estimated using a Taylor expansion:

$$
\delta Y_{\mathrm{i}} \approx \frac{\partial Y_{\mathrm{i}}}{\partial Z} \delta Z+\frac{\partial Y_{\mathrm{i}}}{\partial \mathcal{Y}} \delta \mathcal{Y}+\frac{1}{2}\left[\frac{\partial^{2} Y_{\mathrm{i}}}{\partial Z^{2}}(\delta Z)^{2}+\frac{\partial^{2} Y_{\mathrm{i}}}{\partial \mathcal{Y}^{2}}(\delta \mathcal{Y})^{2}+\frac{\partial^{2} Y_{\mathrm{i}}}{\partial Z \partial \mathcal{Y}} \delta Z \delta \mathcal{Y}\right]
$$

From (14) can be seen that when a thermo-chemical variable is sufficiently smooth, i.e. having small higher order terms, an error in individual $Z$ and/or $\mathcal{Y}$ measurements does not cause a significant error in the averaged value of the thermo-chemical variable since positive and negative errors cancel out if $\delta Z$ and $\delta \mathcal{Y}$ are symmetrically distributed. When the higher order terms in (14) are not negligible, uncertainties in individual $Z$ and/or $\mathcal{Y}$ measurements will cause a non-negligible error in the averaged value of retrieved variables. In Fig. $1, \mathrm{H}_{2} \mathrm{O}$ and $\mathrm{OH}$ are shown in $Z-\mathcal{Y}$ space: $\mathrm{H}_{2} \mathrm{O}$ has negligible higher order terms while for $\mathrm{OH}$ these terms are far from negligible and will likely cause an underestimation of maximum $\mathrm{OH}$ mass fractions and an overestimation of small $\mathrm{OH}$ mass fractions.

In order to determine systematic errors, conditionally (ensemble) averaged predicted mass fractions are presented together with standard deviations of instantaneous measurements. To determine these averaged mass fractions measured mass fractions are clustered in 40 equidistant bins. Bins containing less than 25 data points are not considered in this analysis due to the lack of statistical convergence of these bins. For each instantaneous measurement, corresponding data is retrieved from FGM databases as a function of $Z$ and $\mathcal{Y}$ which are reconstructed from measured mass fractions. Subsequently the data retrieved from FGM databases is averaged bin-wise. This data is then compared to experimental data which is also averaged with the same procedure.

When data is retrieved from the FGM databases the experimentally observed $Z$ is bounded between zero and unity and $\mathcal{Y}$ is bounded to its minimum and maximum values as found in the FGM database. In Fig. 2 it can be seen that around $Z=0.4$ a significant number of super-equilibrium points is observed. In this analysis these super-equilibrium measurements are replaced by corresponding maximum $\mathcal{Y}$ values as found in the FGM database.

$\mathrm{H}_{2} \mathrm{O}$ mass fractions can be predicted very accurately using any type of FGM. To illustrate this, the test cases with the minimum and maximum error, as defined in (13), are shown in Fig. 3. The well predicted averaged value and the negligible standard deviation can be attributed to the small measurement uncertainty for $\mathrm{H}_{2} \mathrm{O}$ and the smooth behavior of $\mathrm{H}_{2} \mathrm{O}$ in $Z-\mathcal{Y}$ space. The only deviation from the $Y_{\mathrm{i}}^{\mathrm{FGM}}=Y_{\mathrm{i}}^{\mathrm{EXP}}$ line can be seen for the maximum measured mass fractions which do not occur in any FGM database. It can be concluded that predictions for $\mathrm{H}_{2} \mathrm{O}$ are rather insensitive to the type of FGM which is used. 

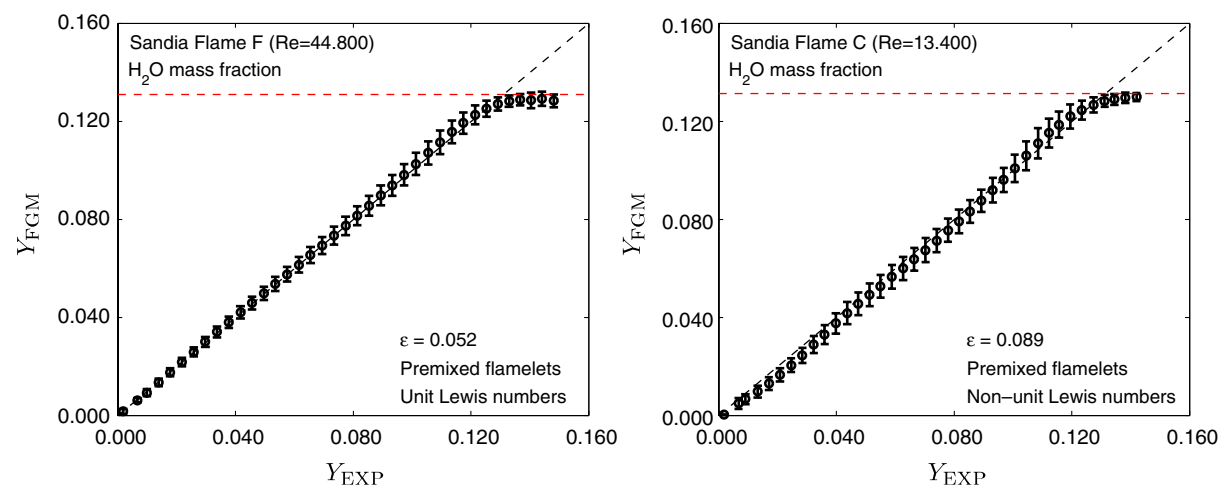

Fig. 3 Conditional averages (circles) together with standard deviations (error bars) of predicted $\mathrm{H}_{2} \mathrm{O}$ mass fractions (vertical axis) versus measured mass fractions. The horizontal dashed red line indicates the maximum $\mathrm{H}_{2} \mathrm{O}$ mass fraction present in the FGM database. The situation with the smallest error is shown on the left, the situation with the largest error is shown on the right. (Color in digital version only)

For $\mathrm{H}_{2}$ mass fractions, shown in Fig. 4, the premixed flamelet-based FGM databases, and especially the FGM-PC, yield a significant overestimation. Standard deviations are substantial and the averaged predicted $\mathrm{H}_{2}$ mass fractions significantly exceeds the measured mass fractions. Both counterflow diffusion flamelet-based FGM databases show smaller standard deviations and the predicted mass fractions correspond well to measured mass fractions. For large measured mass fractions $\left(Y_{\mathrm{H}_{2}}>0.004\right)$ the FGM-DC tends to slightly underestimate $\mathrm{H}_{2}$ mass fractions, this is not observed for the FGM-DL database. The reason the standard deviation is considerable is twofold: first of all the measurement uncertainty for hydrogen equals $10 \%$, in very fuel-rich conditions this uncertainty can rise to $30 \%$ due to interference with hydrocarbons [17]. Second of all the higher order derivatives of $\mathrm{H}_{2}$ in $Z-\mathcal{Y}$ space are not negligible resulting in errors in averaged values and standard deviations.
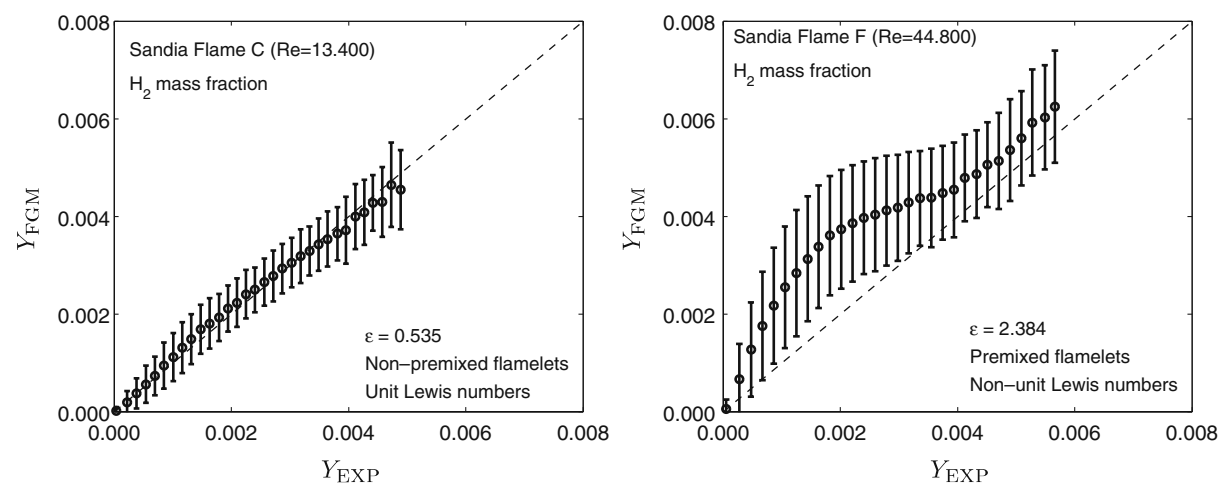

Fig. 4 Conditional averages (circles) together with standard deviations (error bars) of predicted $\mathrm{H}_{2}$ mass fractions (vertical axis) versus measured mass fractions. The horizontal dashed red line indicates the maximum $\mathrm{H}_{2}$ mass fraction present in the FGM database. The situation with the smallest error is shown on the left, the situation with the largest error is shown on the right. (Color in digital version only) 
Scatter plots of measured $\mathrm{CO}_{2}$ mass fractions versus predicted $\mathrm{CO}_{2}$ mass fractions from premixed flamelet-based FGM databases show a two-branch structure, of which one branch shows a significant underestimation of $\mathrm{CO}_{2}$ mass fractions (Fig. 5). The branch exhibiting a significant underestimation of $\mathrm{CO}_{2}$ mass fractions corresponds to fuel-rich combustion and will be discussed more in-depth in Section 3.2. This two-branch structure also causes the conditionally averaged predicted values to be significantly smaller than measured ones. Counterflow diff usion flamelet-based FGM databases yield better predictions for $\mathrm{CO}_{2}$ mass fractions than premixed flameletbased FGM databases: standard deviations are substantially smaller. The FGMDL database shows a better correspondence between the averaged mass fractions than the FGM-DC database: the latter shows a systematic underestimation of conditionally averaged $\mathrm{CO}_{2}$ mass fractions. Measurement uncertainty for $\mathrm{CO}_{2}$ is only $4 \%$ implying that errors mainly originate from reconstruction of $Z$ and $\mathcal{Y}$ and dissimilarities between the measured and tabulated chemistry.

From the averaged values in Fig. 6 can be seen that the FGM-PL database overestimates CO mass fractions; this is also observed for other flames. The FGMPC database exhibits the same trend but to a smaller extent. This overestimation of $\mathrm{CO}$ mass fractions is to be expected because of the underestimation of $\mathrm{CO}_{2}$ mass fractions while the element mass fraction of carbon must be conserved. Counterflow diffusion flamelet-based FGM databases yield more accurate predictions, although small $\mathrm{CO}$ mass fractions $\left(Y_{\mathrm{CO}}<0.05\right)$ tend to be slightly overestimated while large $\mathrm{CO}$ mass fractions $\left(0.05<Y_{\mathrm{CO}}<0.085\right)$ tend to be underestimated. Substantial standard deviations are partly caused by the measurement uncertainty for $\mathrm{CO}$ which equals $10 \%$ and in very fuel-rich conditions can rise to $20 \%$.

For both the premixed- and counterflow diffusion flamelet-based databases it was observed that a multi-component diffusion transport model yields reduced $\mathrm{OH}$ mass fractions when compared to databases with a unit Lewis number transport model. Since $\mathrm{OH}$ is assumed to be in quasi steady state the reduction of the maximum mass
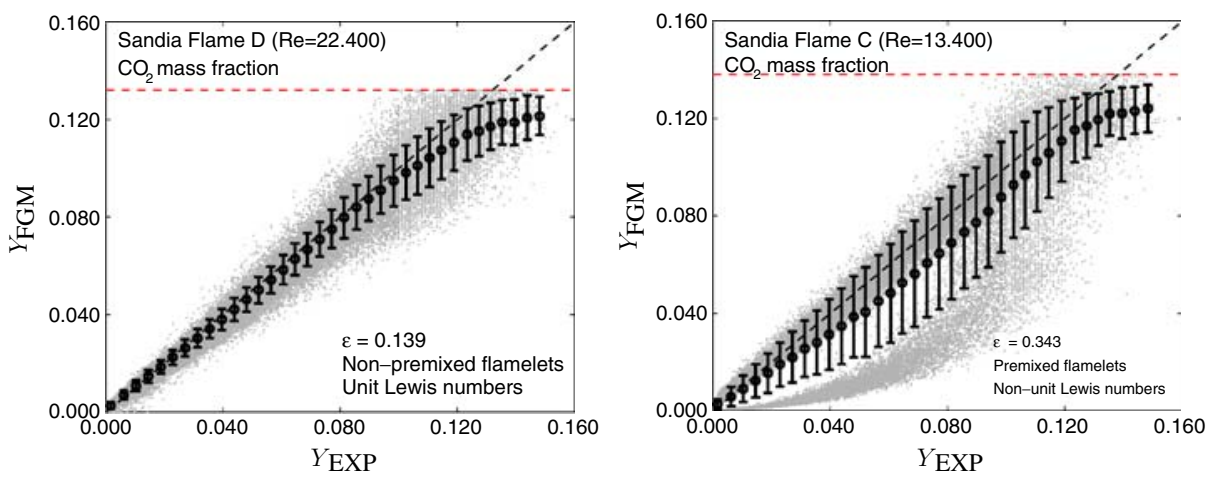

Fig. 5 Conditional averages (circles) together with standard deviations (error bars) of predicted $\mathrm{CO}_{2}$ mass fractions (vertical axis) versus measured mass fractions. The horizontal dashed red line indicates the maximum $\mathrm{CO}_{2}$ mass fraction present in the FGM database. The situation with the smallest error is shown on the left, the situation with the largest error is shown on the right. Instantaneous measurements are shown to illustrate the two-branch structure of predictions from premixed flamelet-based FGM databases. (Color in digital version only) 

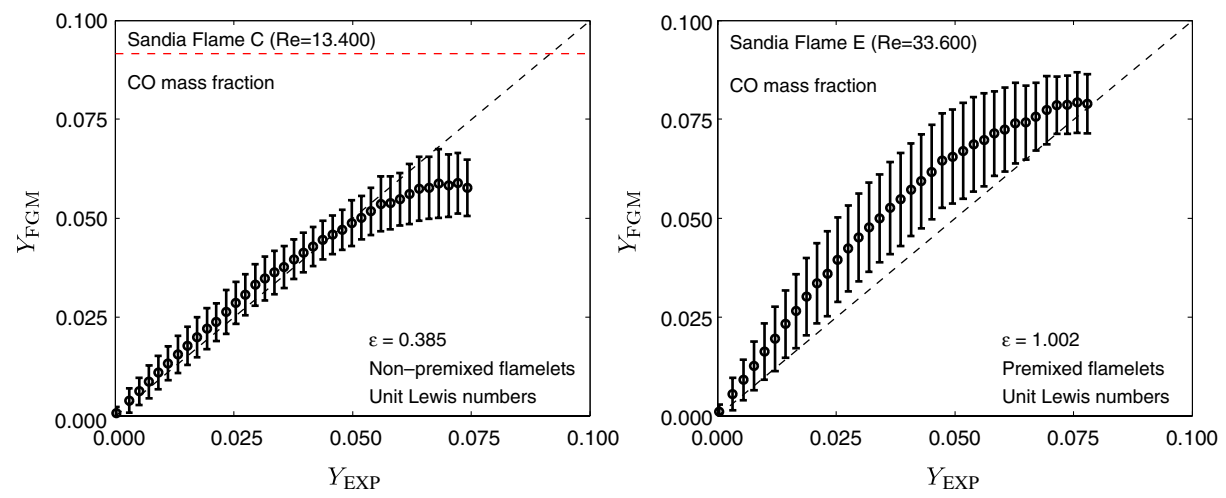

Fig. 6 Conditional averages (circles) together with standard deviations (error bars) of predicted CO mass fractions (vertical axis) versus measured mass fractions. The horizontal dashed red line indicates the maximum $\mathrm{CO}$ mass fraction present in the FGM database. The situation with the smallest error is shown on the left, the situation with the largest error is shown on the right. (Color in digital version only)

fraction (Fig. 7), when a multi-component diffusion transport model is used instead of a unit Lewis number transport model, is most probably caused by differential diffusion of other species. For all FGM databases standard deviations are very large but for the FGM-PL, the FGM-PC and the FGM-DL databases can be seen that the conditionally averaged predicted $\mathrm{OH}$ mass fractions correspond well to the conditionally averaged measured $\mathrm{OH}$ mass fractions for $Y_{\mathrm{OH}}<0.004$. For large $\mathrm{OH}$ mass fractions $\left(Y_{\mathrm{OH}}>0.004\right)$ the averaged mass fractions are underestimated; this can again be attributed to the fact that maximum measured mass fractions do not occur in the FGM database. The FGM-DC also exhibits this underestimation at large $\mathrm{OH}$ mass fractions, in addition small $\mathrm{OH}$ mass fractions $\left(Y_{\mathrm{OH}}<0.0025\right)$ are overestimated. The large standard deviations are caused by the uncertainty in experiments which serve as input, and a very localized $\mathrm{OH}$ peak in $Z-\mathcal{Y}$ space as can be seen in Fig. 1.

From the results above and the overview of errors in Table 2 it can be concluded that counterflow diffusion flamelet-based databases yield significant better predictions than premixed flamelet-based ones. Premixed flamelet-based databases tend to overestimate $\mathrm{H}_{2}$ and $\mathrm{CO}$ mass fractions while underestimating $\mathrm{CO}_{2}$ mass fractions. Comparison of results from the FGM-DL and the FGM-DC database shows that the FGM-DL yields more accurate results for the conditionally averaged species mass fractions. For OH mass fractions the FGM-PL, the FGM-PC and the FGM-DL database yield comparable results, both in conditionally averaged $\mathrm{OH}$ mass fractions and standard deviations. The FGM-DC database yields less accurate results for the conditionally averaged $\mathrm{OH}$ mass fractions than the other databases. For water the choice of FGM database has no significant influence on accuracy of predictions.

\subsection{Averaged mass fractions conditioned on $Z$}

In the previous section predictive performance of the four different FGM databases, as stated in Table 1, has been globally assessed for the Sandia Flames C to F. It was 

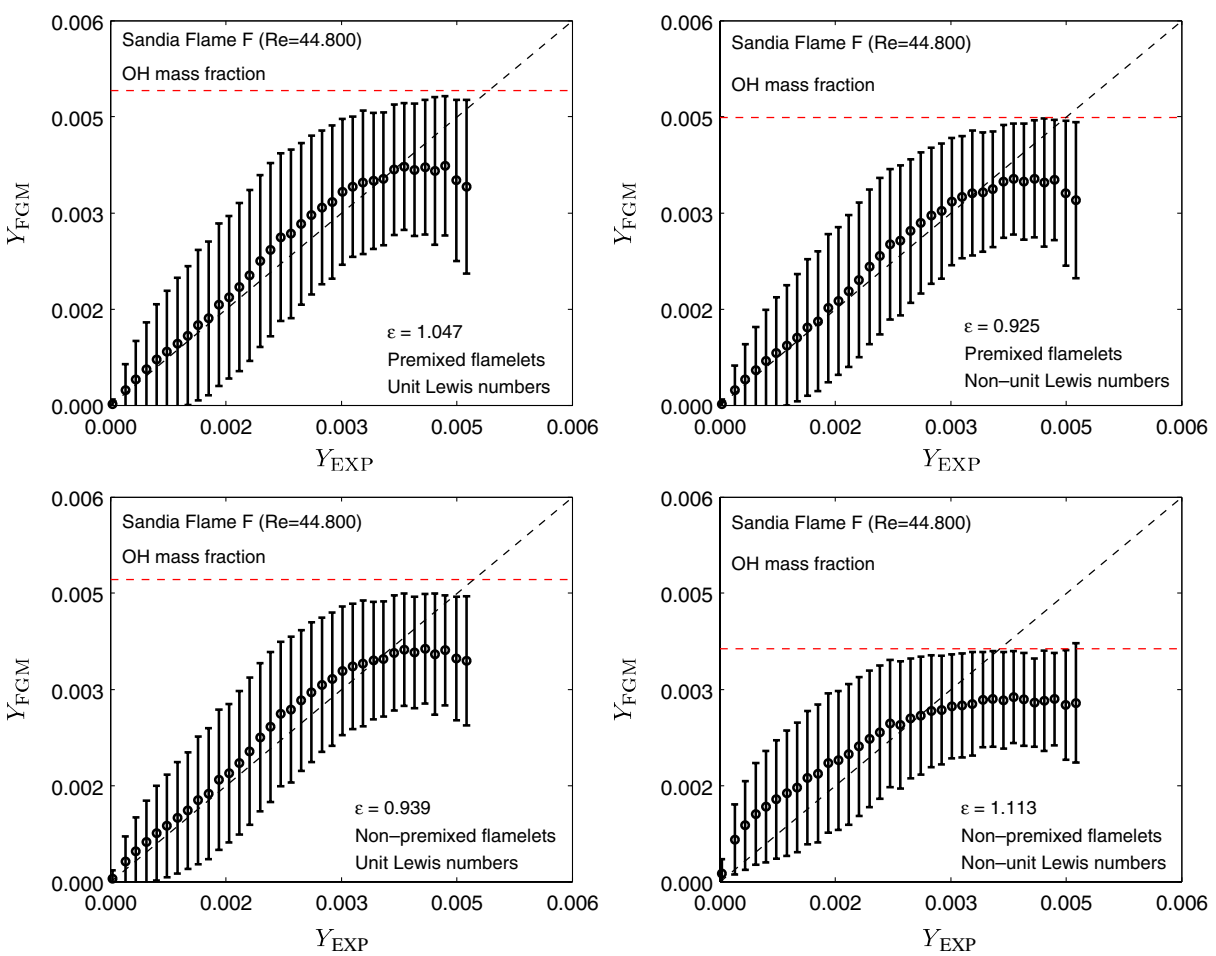

Fig. 7 Conditional averages (circles) together with standard deviations (error bars) of predicted $\mathrm{OH}$ mass fractions (vertical axis) versus measured mass fractions. The horizontal dashed red line indicates the maximum $\mathrm{OH}$ mass fraction present in the FGM database. (Color in digital version only)

observed that for $\mathrm{H}_{2}, \mathrm{CO}_{2}$ and $\mathrm{CO}$ the FGM-PL and the FGM-PC database cannot represent observed mass fractions accurately. The counterflow diffusion flameletbased FGM databases perform significantly better, especially the FGM-DL database. In this section predicted mass fractions will be compared to experimentally observed mass fractions for several heights in Flames $\mathrm{C}$ to $\mathrm{F}$. Results will be presented conditioned on $Z$ in order to gain more insight in underlying physical phenomena. Experimental data, $\mathcal{O}\left(10^{5}\right)$ instantaneous measurements per height, are clustered in 40 bins in $Z$-direction. The procedure is similar to the one described in Section 3.1 but now only bins containing only 10 data points or less are discarded.

It was already seen in Fig. 4 that the FGM-PL and the FGM-PC database significantly overestimate $\mathrm{H}_{2}$ mass fractions; from Fig. 8 it is clearly visible that

Table 2 Maximum error as defined by (13) for different species and FGM databases

\begin{tabular}{llllll}
\hline & $\mathrm{H}_{2} \mathrm{O}$ & $\mathrm{H}_{2}$ & $\mathrm{CO}_{2}$ & $\mathrm{CO}$ & $\mathrm{OH}$ \\
\hline FGM-PL & 0.056 & 1.36 & 0.273 & 1.00 & 1.48 \\
FGM-PC & 0.089 & 2.38 & 0.343 & 0.769 & 1.34 \\
FGM-DL & 0.061 & 0.686 & 0.147 & 0.545 & 1.27 \\
FGM-DC & 0.070 & 0.833 & 0.237 & 0.784 & 1.41 \\
\hline
\end{tabular}



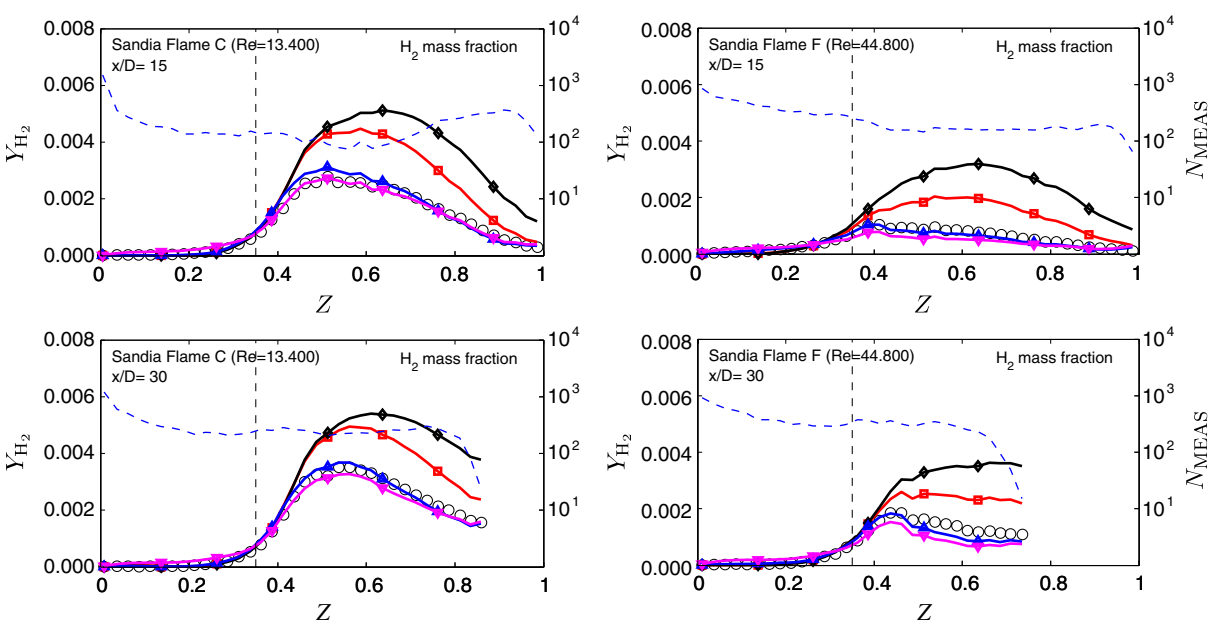

Fig. 8 Averaged conditional $\mathrm{H}_{2}$ mass fractions in Sandia Flame $\mathrm{C}$ and $\mathrm{F}$ at $\mathrm{x}=15 \mathrm{D}$ and 30D. Black circles denote measurements, red lines with square markers denote predictions from the FGM-PL, black lines with diamond markers denote predictions from the FGM-PC, blue lines with upwards directing triangle markers denote predictions from the FGM-DL and magenta lines with downwards directing triangle markers denote predictions from the FGM-DC. The blue dashed line represents the number of measurements per $Z$-bin; values are stated on the right y-axis. The vertical black dashed line indicates the stoichiometric mixture fraction. (Color in digital version only)

this overestimation occurs under fuel-rich conditions. This can be explained by the fact that species can only diffuse in $\mathcal{Y}$-direction in premixed flamelets. In nonpremixed flamelets these species diffuse towards the $Z=Z_{\text {stoich }}$ plane where they are further oxidized [15]. The counterflow diffusion flamelet-based databases both yield substantially more accurate predictions for $\mathrm{H}_{2}$ mass fractions. Apparently the underestimation of $\mathrm{H}_{2}$ mass fractions by the FGM-DC at large $\mathrm{H}_{2}$ mass fraction values, as can be seen in Fig. 4, does not have a visible effect on spatial predictions.

As shown in Fig. 9, the CO mass fraction tends to be overestimated by premixed flamelet-based databases, although not as serious as $\mathrm{H}_{2}$ mass fractions. The FGM-PL yields a larger overestimation of CO mass fractions than the FGM-PC, especially in fuel-rich regions $(Z>0.5)$. The FGM-DC can be seen to overestimate $\mathrm{CO}$ mass fractions in fuel-lean areas. Similar profiles are found at other heights. At each height the observation can be made that the maximum $\mathrm{CO}$ mass fractions decreases with increasing Reynolds number. This phenomena is also observed for counterflow diffusion flamelets with increasing strain rate; on the hand it should be verified whether the steady-state assumption, implied by the use of a tabulation method, holds for CO. The overprediction of CO mass fractions by the FGM-PL in fuel-rich regions $\left(Z>Z_{\text {st }}\right)$ and by the FGM-DC in fuel-lean regions $\left(Z<Z_{\text {st }}\right)$ corresponds to an underprediction of $\mathrm{CO}_{2}$ mass fractions in the same regions.

For $\mathrm{OH}$ it is observed that FGM-PL, the FGM-PC and the FGM-DL database practically predict the same $\mathrm{OH}$ mass fractions. However, the maximum $\mathrm{OH}$ mass fractions are overestimated by $25 \%$ at maximum. The comparison between the FGM-DC and FGM-DL shows a remarkable phenomena: near the inlet plane $(\mathrm{x} / \mathrm{D}=7.5$ and $\mathrm{x} / \mathrm{D}=15)$ the FGM-DC predicts a lower maximum $\mathrm{OH}$ mass fraction than the FGM-DL but this maximum is located at approximately the same $Z$ as 

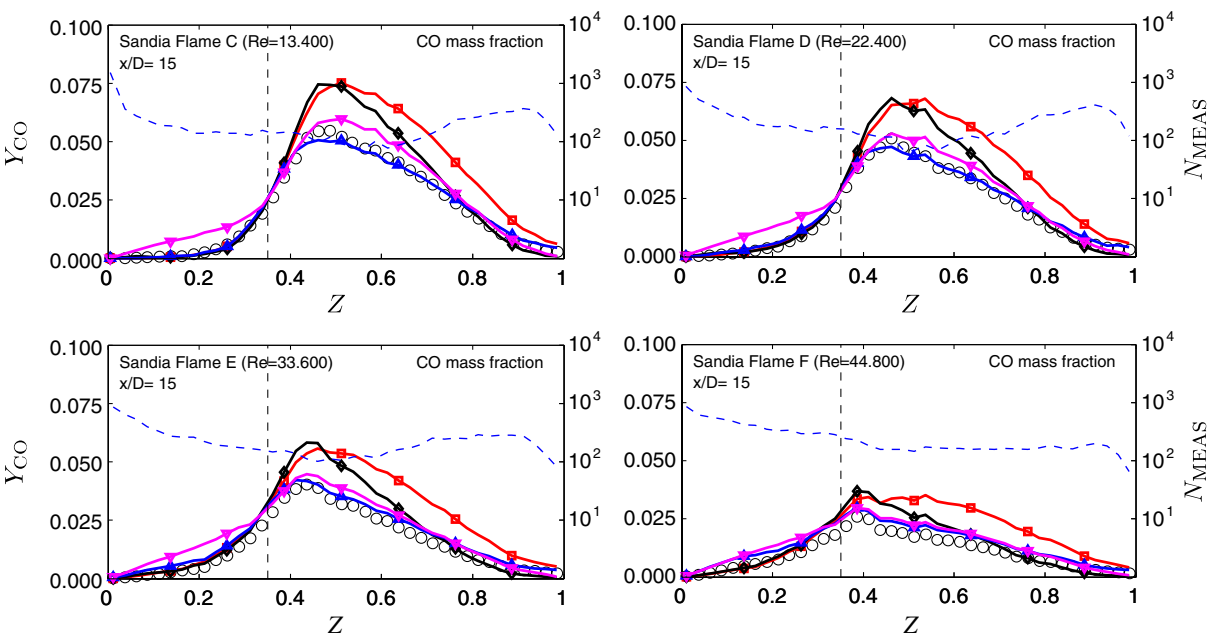

Fig. 9 Averaged conditional CO mass fractions in Sandia Flame C, D, E and F at x =15D. Symbols identical to those in Fig. 8 have been used. (Color in digital version only)

where the maximum $\mathrm{OH}$ mass fraction is predicted by the FGM-DL. In the far-field $(\mathrm{x} / \mathrm{D}=45$ and $\mathrm{x} / \mathrm{D}=60)$ the maximum $\mathrm{OH}$ mass fractions are similar for both FGM databases but the maximum $\mathrm{OH}$ mass fraction predicted by the FGM-DC is shifted to more fuel-lean zones. Figure 10 shows these trends for Sandia Flame D; Flame C, $\mathrm{E}$ and $\mathrm{F}$ show a similar trend. No exact reason can be given for these phenomena but it is believed by the authors that differential diffusion of other species causes an
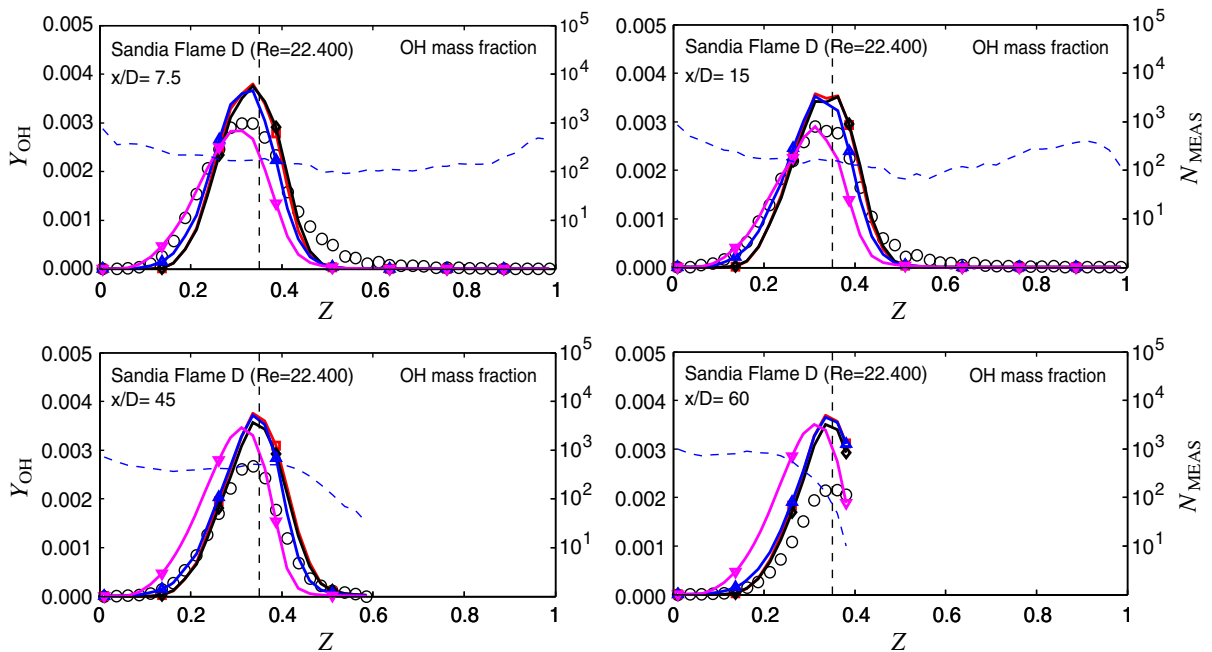

Fig. 10 Reynolds-averaged conditional $\mathrm{OH}$ mass fractions in Sandia Flame $\mathrm{D}$ at $\mathrm{x}=7.5 \mathrm{D}, 15 \mathrm{D}, 45 \mathrm{D}$ and 60D. Symbols identical to those in Fig. 8 have been used. (Color in digital version only) 
alteration in their concentrations and thereby an alteration in the production and consumption rate of $\mathrm{OH}$.

Since $\mathrm{OH}$ mass fractions tend to increase with increasing temperature it was put forward that the overestimation of $\mathrm{OH}$ might be linked to an overestimation of temperature by FGM databases. An analysis for the FGM-DL database shows that temperature is slightly underestimated, within the measurement uncertainty equal to $3 \%$ [17], for heights up to $x / D=45$ for all flames. When temperature is added as a control variable, i.e. mapping is now described by $\phi=\phi(Z, \mathcal{Y}, T)$, OH predictions do not improve and sometimes even worsen. The addition of temperature as a third control variable is therefore abandoned.

\subsection{Differential diffusion effects}

From previous results for $\mathrm{H}_{2}$ and $\mathrm{CO}$ can be concluded that diffusion in $Z$-direction is of major importance for the Sandia Flames considered here. The accuracy of reproduction of differential diffusion phenomena will therefore be assessed for counterflow diffusion flamelet-based databases only. To compare the FGM-DL to the FGM-DC the differential diffusion parameter $z$ as defined by Barlow et al. [15] is used to quantify the relative importance of molecular diffusion compared to turbulent transport. The definition of the differential diffusion parameter reads:

$$
z=\frac{\left[\mathcal{Z}_{H}-\mathcal{Z}_{H, 2}\right]}{\left[\mathcal{Z}_{H, 1}-\mathcal{Z}_{H, 2}\right]}-\frac{\left[\mathcal{Z}_{C}-\mathcal{Z}_{C, 2}\right]}{\left[\mathcal{Z}_{C, 1}-\mathcal{Z}_{C, 2}\right]}
$$

in which the same denotations have been used as in (2).

In Fig. 11 the differential diffusion parameter predicted by the FGM-DL and FGM-DC databases is compared to the experimentally observed differential diffusion parameter for Flame $\mathrm{C}$ at multiple heights. In Flame $\mathrm{C}$, having only a moderate turbulence intensity, close to the inlet plane $(x=7.5 \mathrm{D}$ and $\mathrm{x}=15 \mathrm{D})$ the FGM-DC database yields more accurate predictions for the differential diffusion parameter than the FGM-DL database. With increasing axial coordinate the FGM-DL database yields better results than the FGM-DC database.

In Fig. 12 the differential diffusion parameter predicted by the FGM-DL and FGM-DC databases is compared to the experimentally observed differential diffusion parameter close to the inlet plane $(\mathrm{x} / \mathrm{D}=7.5)$. For all flames the decrease of $z$ for $Z \in[0.35,0.8]$, caused by the preferential diffusion of $\mathrm{H}_{2}$ away from this fuel-rich side of the stoichiometric condition [15], is severely overestimated by the FGM-DC; here the FGM-DL corresponds significantly better to measurements. For Sandia Flame C it can be observed that the FGM-DC in general yields the best results although for the region $Z \in[0.6,1]$ the FGM-DL corresponds slightly better to measurements. This can be explained by the fact that this fuel-rich zone corresponds to an area close to the fuel jet where turbulent mixing is important. To illustrate this the turbulent kinetic energy $k$, which is reconstructed from data by Schneider et al. [25] is shown in the same figure. Reconstruction of $k$ makes use of the assumption that fluctuations in tangential direction are comparable to fluctuations in radial direction: $k=\frac{1}{2}\left(\overline{u^{\prime} u^{\prime}}+2 \overline{v^{\prime} v^{\prime}}\right)$. Turbulent fluctuations are only available at all heights for Flame D and F. From Figs. 12 and 13 a significant relation can be observed between the increase of turbulent kinetic energy and a shift from the FGM-DC database to the FGM-DL database. These trends have also been observed 

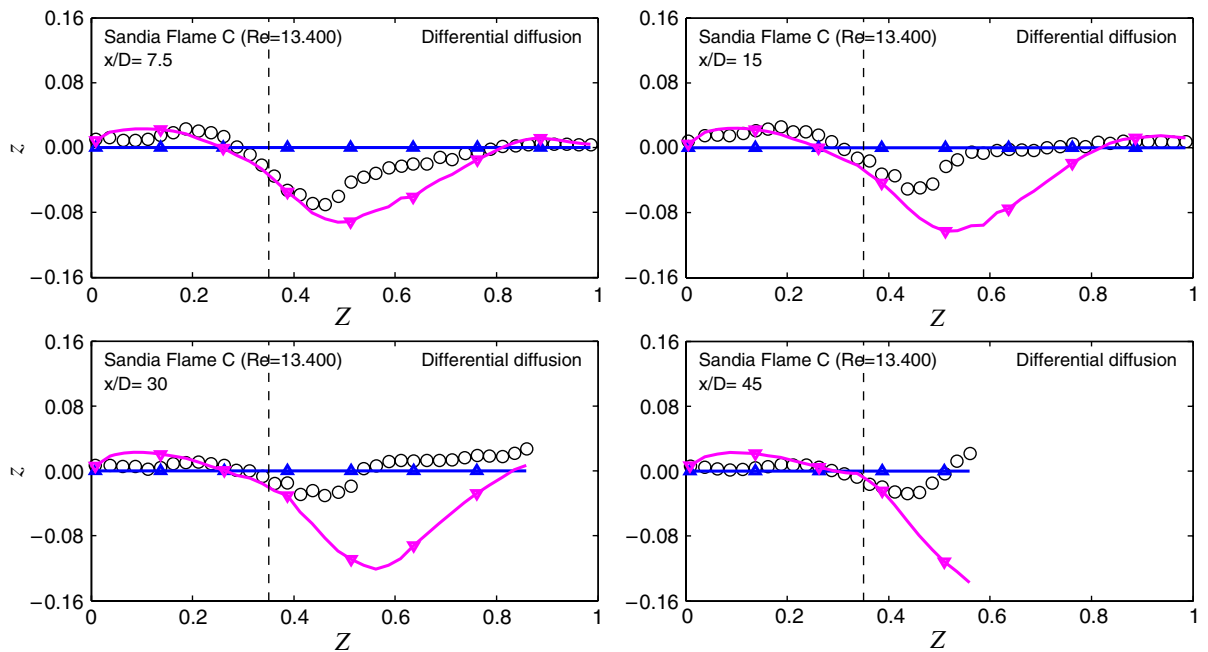

Fig. 11 Differential diffusion parameter $z$ in Sandia Flame $C$ at $x=7.5 \mathrm{D}, \mathrm{x}=15 \mathrm{D}, \mathrm{x}=30 \mathrm{D}$ and $\mathrm{x}=45 \mathrm{D}$. Black circles denote measurements, blue lines with upwards directing triangle markers denote predictions from the FGM-DL and magenta lines with downwards directing triangle markers denote predictions from the FGM-DC. The black dashed line indicates the stoichiometric mixture fraction. (Color in digital version only)

by Barlow [15]; this study confirms these observations by comparison with a FGMDL and a FGM-DC database. Additionally it is showed that the departure from the FGM-DC database towards the FGM-DL database for the region $Z \in[0.6,1]$ is most likely caused by turbulent mixing between the fuel jet and the co-flow.
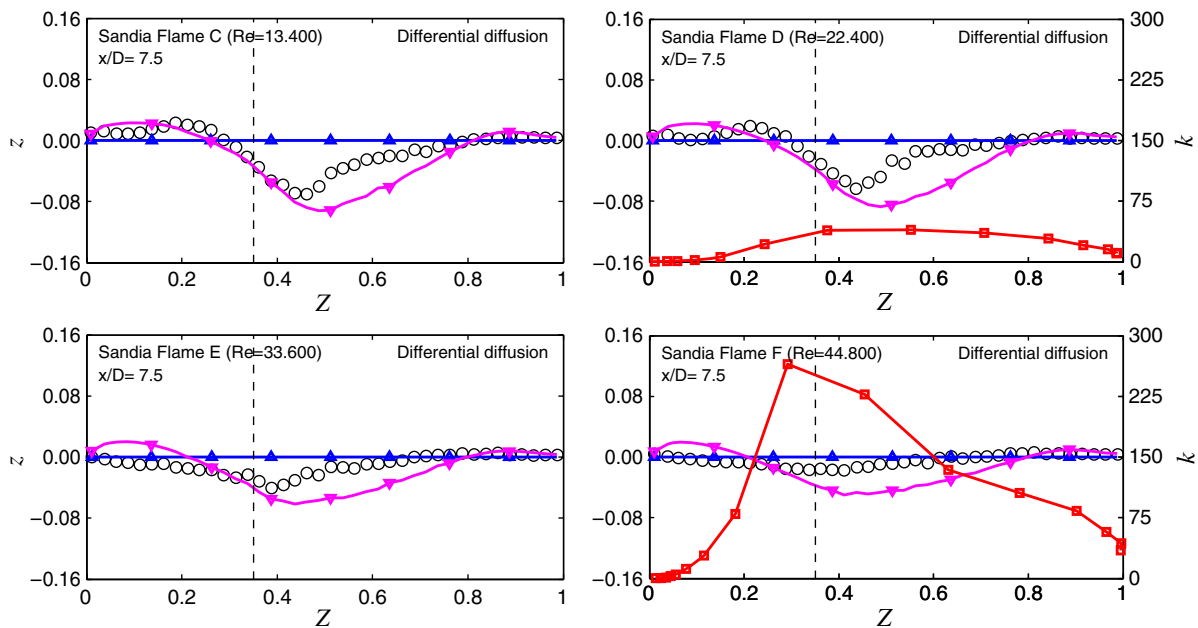

Fig. 12 Differential diffusion parameter $z$ in Sandia Flame C, D, E and F at $\mathrm{x}=7.5 \mathrm{D}$. Symbols identical to those in Fig. 11 have been used. In addition the red line with square markers indicates the reconstructed turbulent kinetic energy. (Color in digital version only) 
Fig. 13 Differential diffusion parameter $z$ in Sandia Flame $\mathrm{D}$ at $\mathrm{x}=15 \mathrm{D}$ together with reconstructed turbulent kinetic energy. Symbols identical to those in Fig. 12 have been used. (Color in digital version only)

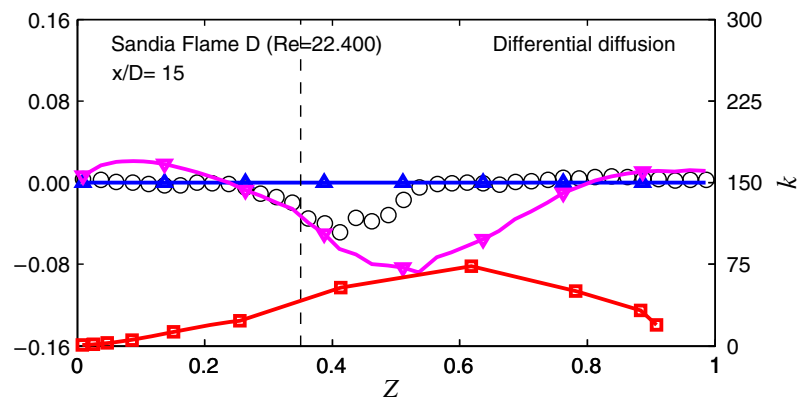

\section{Conclusion}

In this study the accuracy of FGM databases in predicting $\mathrm{H}_{2} \mathrm{O}, \mathrm{H}_{2}, \mathrm{CO}_{2}, \mathrm{CO}$ and $\mathrm{OH}$ mass fractions in partially-premixed jet flames has been assessed. Four FGM databases have been compared: databases using premixed or counterflow diffusion flamelets either with multi-component diffusion or unit Lewis numbers for all species. Both counterflow diffusion flamelet-based databases proved to be significantly more accurate than premixed-based ones for $\mathrm{H}_{2}, \mathrm{CO}_{2}$ and $\mathrm{CO}$ mass fraction predictions. For fuel-rich conditions the FGM-PL and FGM-PC database tend to severely overestimate $\mathrm{H}_{2}$ and $\mathrm{CO}$ mass fractions while underestimating $\mathrm{CO}_{2}$ mass fractions. Differential diffusion effects are most visible in Flame C and D and only close to the inlet plane. An increase in turbulent kinetic energy generated in the shear layer between the fuel jet and the co-flow causes the transition from a multi-component diffusion behavior to unit Lewis number diffusion. From results presented in Section 3 can be concluded that an FGM-DL database, parameterized on $Z$ and $\mathcal{Y}$, can accurately predict $\mathrm{H}_{2} \mathrm{O}, \mathrm{H}_{2}, \mathrm{CO}_{2}$ and $\mathrm{CO}$ mass fractions for the Sandia Flames $\mathrm{C}$ to F. For OH the FGM-DL database yields a good correspondence between averages of large ensembles of measured and predicted values but for individual measurements the standard deviation is large due to measurement uncertainties $(10 \%)$ and reconstruction uncertainties for $Z$ and $\mathcal{Y}$ in combination with a very localized $\mathrm{OH}$ peak in $Z-\mathcal{Y}$ space as can be seen in Fig. 1 . When averaged $\mathrm{OH}$ mass fractions are conditioned on $Z$, the FGM-DL predicts the location of maximum $\mathrm{OH}$ mass fraction in $Z$-space accurately. However, the $\mathrm{OH}$ mass fraction at its maximum is overpredicted by approximately $25 \%$.

The observed error in results from RANS and LES simulations using FGM (or FPI) databases therefore must stem rather from the subgrid model and modeling assumptions in the transport equations than from combustion chemistry representation by an FGM database provided that the choice of flamelet type and molecular transport model agree with the modeled flame. These conclusions are not surprising or contradictory to previous results but the accuracy that can be reached with a twodimensional $(Z, \mathcal{Y})$ FGM-DL database is striking. Even for Sandia Flame F, which is known to exhibit significant extinction and re-ignition events, the predictions are in close agreement with experimental observations.

Acknowledgements This work received funding from the European Community through the project TIMECOP-AE (project AST5-CT-2006-030828). It reflects only the authors' views and the Community is not liable for any use that may be made of the information contain therein. 
Open Access This article is distributed under the terms of the Creative Commons Attribution Noncommercial License which permits any noncommercial use, distribution, and reproduction in any medium, provided the original author(s) and source are credited.

\section{References}

1. Pope, S.B.: Combust. Theory Model. 1, 41-63 (1997)

2. Maas, U., Pope, S.B.: Combust. Flame 88, 239-264 (1992)

3. Bykov, V., Maas, U.: Combust. Theory Model. 1, 1-2 (2006)

4. Bykov, V., Maas, U.: Proc. Combust. Inst. 31, 465-472 (2007)

5. Gicquel, O., Darabiha, N., Thevenin, D.: Proc. Combust. Inst. 28, 1901-1908 (2000)

6. van Oijen, J.A., de Goey, L.P.H.: Combust. Sci. Technol. 161, 113-137 (2000)

7. Peters, N.: Prog. Energy Combust. Sci. 10, 319-339 (1984)

8. Chen, J.-Y., Dibble, R.W.: Combust. Sci. Technol. 84, 45-50 (1992)

9. Pitsch, H., Steiner, H.: Phys. Fluids 12(10), 2541-2554 (2000)

10. Vervisch, L., Hauguel, R., Domingo, P., Rulland, M.: J. Turbul. 5(4), 1-36 (2004)

11. Fiorina, B., Gicquel, O., Vervisch, L., Carpentier, S., Darabiha, N.: Combust. Flame 140, 147-160 (2005)

12. Vreman, A.W., Albrecht, B.A., van Oijen, J.A., de Goey, L.P.H., Bastiaans, R.J.M.: Combust. Flame 153, 394-416 (2008)

13. Barlow, R.S., Frank, J.: Piloted $\mathrm{CH}_{4}$ /air flames C, D, E and F-release 2.0. Technical report, Sandia National Laboratories (2003)

14. Bilger, R.W.: Combust. Flame 80, 135-149 (1990)

15. Barlow, R.S., Frank, J.H., Karpetis, A.N., Chen, J.-Y.: Combust. Flame 143, $433-449$ (2005)

16. Barlow, R.S., Frank, J.H.: Proc. Combust. Inst. 27, 1087-1095 (1998)

17. Barlow, R.S., Karpetis, A.N., Frank, J.H., Chen, J.-Y.: Combust. Flame 127, 2102-2118 (2001)

18. de Goey, L.P.H., Ten Thije Boonkkamp, J.H.M.: Combust. Sci. Technol. 122, 399-405 (1997)

19. Dixon-Lewis, G.: Proc. Combust. Inst. 23, 305-324 (1990)

20. Somers, L.M.T.: Ph.D. thesis, Eindhoven University of Technology (1994)

21. Smith, G.P., Golden, D.M., Frenklach, M., Moriarty, N.W., Eiteneer, B., Goldenberg, M., Bowman, C.T., Hanson, R.K., Song, S., Gardiner, W.C. Jr., Lissianski, V.V., Qin, Z.: Grimech 3.0 reaction mechanism. Technical report, Sandia National Laboratories (2000)

22. Liu, K., Pope, S.B., Caughey, D.A.: Combust. Flame 141, 89-117 (2005)

23. Carter, C.D., Barlow, R.S.: Opt. Lett. 19, 299-301 (1994)

24. Nguyen, Q.V., Dibble, R.W., Carter, C.D., Fiechtner, G.J., Barlow, R.S.: Combust. Flame 105, 499-510 (1996)

25. Schneider, Ch., Dreizler, A., Janicka, J., Hassel, E.P.: Combust. Flame 135, 185-190 (2003) 